\title{
Motion Analysis System for Robot Traction Device Evaluation and Design
}

\author{
Scott J. Moreland ${ }^{1}$, Krzysztof Skonieczny ${ }^{1}$ and, David S. Wettergreen ${ }^{1}$ \\ ${ }^{1}$ Robotics Institute, Carnegie Mellon University, Pittsburgh, PA, USA.
}

\begin{abstract}
Though much research has been conducted regarding traction of tires in soft granular terrain, little empirical data exist on the motion of soil particles beneath a tire. A novel experimentation and analysis technique has been developed to enable detailed investigation of robot interactions with granular soil. This technique, the Shear Interface Imaging Analysis method, provides visualization and analysis capability of soil shearing and flow as it is influenced by a wheel or excavation tool. The method places a half-width implement (wheel, excavation bucket, etc.) of symmetrical design in granular soil up against a transparent glass sidewall. During controlled motion of the implement, high-speed images are taken of the sub-surface soil, and are processed via optical flow software. The resulting soil displacement field is of very high fidelity and can be used for various analysis types. Identification of clusters of soil motion, shear interfaces and shearing direction/magnitude allow for analysis of the soil mechanics governing traction. The Shear Interface Imaging Analysis Tool enables analysis of robot-soil interactions in richer detail than possible before. Prior state-of-art technique relied on longexposure images that provided only qualitative insight, while the new processing technique identifies sub-millimeter gradations in motion and can do so even for high frequency changes in motion. Results are presented for various wheel types and locomotion modes: small/large diameter, rigid/compliant rim, grouser implementation, and push-roll locomotion.
\end{abstract}

\section{Introduction}

The mobility systems of past and current planetary surface exploration missions are still limited by terrain encountered, demonstrating the need for continued development of traction devices for robotic vehicles. Targets, even whole regions, of scientific interest exist in terrain that is frequently beyond the capability of all flown mobility platforms resulting in the loss of potential scientific return. Of specific challenge on the Moon and Mars are flat ground and slopes covered by loose, low strength regolith. Wheeled mobility systems can become entrenched in these terrains due to excessive slip and sinkage. With the continued exploration of Mars and other planetary bodies the study of wheel-soil behavior in loose, granular material remains imperative for achieving future scientific discoveries. Methods available utilizing common terramechanics approaches do not achieve high fidelity results, especially for non-idealized wheels with complex rim-soil surface inter- 
action. Additionally, soil mechanics processes are not well understood for lightweight vehicles with wheels operating in loose, granular materials where significant soil flow is present.

The design of traction devices such as wheels for planetary rovers rarely involves the detailed analysis of soil shearing and failure patterns. The stress applied to the soil mass is the result of both external loading and the operating state of the wheel-soil system. The 'shear interface' are failure planes that develop in the soil below the region of interaction with the rim (Fig. 3). Shear interfaces can indicate the soil failure type, thrust/motion resistance processes and important soil flows present. Geometry of the rim, presence of grousers, wheel stiffness, contact shape and many other properties have a large effect on soil shearing during operation of a wheel. The performance of a traction device in loose, granular soil is ultimately governed by the soil properties and the shear failures that occur.

As examples, a rotating wheel may induce a forward flow leading to motion resistance and energy loss. Furthermore, a small diameter rigid wheel will cause a rearward flow, forcing particles deep into the soil and then back up again in a " $\mathrm{v}$ "like shape terminating at the rim exit point. In contrast, a footpad utilized by vehicles with walking locomotion generates a significantly different mode of soil failure, which has a shear interface extending well beyond the wheel confinement. The distinct modes of soil failure and flow processes occurring provide insight into the development of traction of broad range of traction devices. Minute details of the wheel rim geometry or mechanics of the wheel carcass have a profound affect on the shearing processes. Geometric features such as the commonly implemented grousers are not able to be incorporated into existing terramechanic modeling techniques, thus inadequate results and misleading representations of the shearing processes are produced by most methods.

This paper will (1) discuss the importance of the investigation of sub-surface soil shearing of traction devices in terramechanic evaluation, (2) introduce a method of analysis called Shear Interface Imaging Analysis [Moreland11b] and (3) by use of examples, discuss the affect commonly considered wheel properties have on soil behavior utilizing the proposed analysis method.

In effort to directly investigate the soil shearing processes occurring due to wheel operation, an experimental approach was taken for this body of work. The study of the principles of traction was investigated by soil behavior analysis rather than bulk performance. The development of a technique we call "Shear Interface Imaging Analysis (SIIA)" was undertaken in order to measure the location and characteristics of the soil shearing in great detail. This method relies on the use of photographing soil grains through a glass-walled bin as a traction device operates. The SIIA technique has proven to produce accurate results and allowed for indepth investigation of unconventional locomotion modes such as push-roll [Moreland11b] and various wheel types for planetary surface vehicles [Moreland11a]. 


\section{Shear Interface Imaging Analysis}

Prior efforts have been made to image soil effects due to travel of a wheel [Bekker60][Wong67] and operation of other devices such as excavation implements [Harrison73]. These researchers produced extensive results, but a limited amount of this work can provide direct insight into the application of design for planetary mobility systems. Previous works focused on validation of existing terramechanic theory, however the analytical models of interest are limited to simplified wheels and soil interaction. As such, this limited the experimentation space to simple/idealized wheels (rigid, wide, no grousers, low slip ratios) and without an investigation of soil behaviors due to a wide range of common design parameters. Existing techniques of soil imaging also produced low fidelity results, were not quantitative and could not assess time varying responses present in the soil. Results in this work show this often does not produce observable shearing processes similar to that of a rolling wheel. Previous imaging work concluded that terramechanic theory does not well represent flow of soil and that similar experimentation methods should be utilized for model development and validation of design [Wong67]. This further motivates the work being conducted utilizing the novel Shear Interface Imaging Analysis method.

Prior methods of imaging relied on long exposure of film to indicate the shear interface. This produced low precision results that could only indicate whether soil was either moving or static and a course average of direction; no other information could be measured. The shear interface imaging analysis technique described in this work is a new method that is capable of recording many types of soil shearing information at high precision. Other techniques for lower precision visualization of soil motion have included tracking tracer particles exposed to UV light [Raschke96], observing changes to a grid pattern of different-colored particles [Khot07], and applying white light speckle autocorrelation to an arrangement of natural and colored sand grains [Gachet03].

\subsection{Description of Technique: Hardware and Software}

The experimental apparatus constructed to analyze the soil shearing below a wheel consists of a glass-walled soil bin filled with regolith simulant, a traction device specimen, an actuated horizontal axis of motion (Fig. 1) and a high-speed camera. The wheel module (Fig. 2) of the imaging test bed is position or velocity controlled in coordination with the horizontal axis to create a commanded, constant slip ratio as the wheel travels forward. A linear rail allows the wheel to translate freely in the vertical direction allowing for natural sinkage to occur and payload weight to be transmitted. A 6-d.o.f. force/torque sensor is incorporated to measure the reaction loads, specifically in the travel direction as a result of traction generated. Sinkage is measured via an optical encoder affixed to the vertical free linear axis. All telemetry; wheel angular velocity, travel velocity, slip, sinkage, load and power are logged simultaneously at $20 \mathrm{~Hz}$ or higher. 


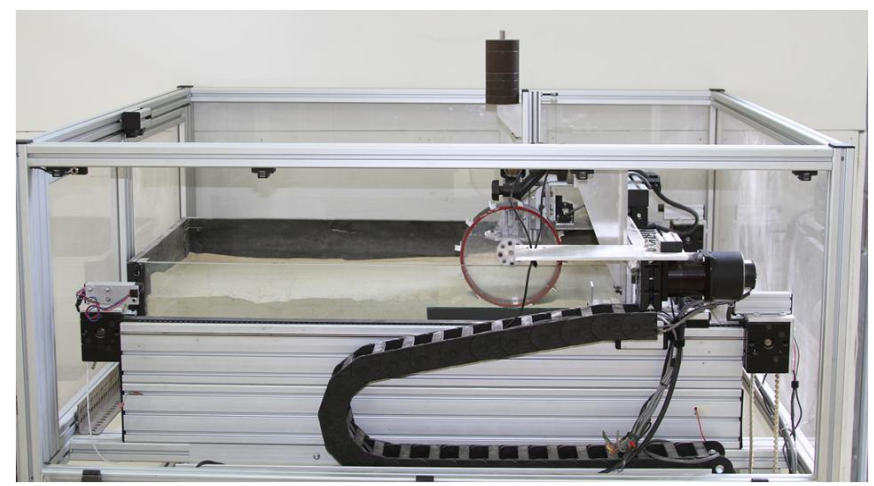

Fig 1: Single Wheel Soil Imaging testbed. Wheel travels from left to right with controlled slip along a belt-driven linear axis.

The wheel for all shear imaging analysis experiments is pressed against a sheet of tempered glass that extends the depth of the soil bin $(27 \mathrm{~cm}$ depth for rig shown). Of importance is the use of a wheel of half the width of the actual specimen of interest and the application of half the payload weight. This aspect has been relied upon for over 50 years [Bekker48] and it has been experimentally validated that if shear stress between glass and soil is negligible, the glass surface acts as a plane of symmetry and the soil behaves as it would directly below an implement twice as wide due to the same boundary conditions [Wong66][Wong67]

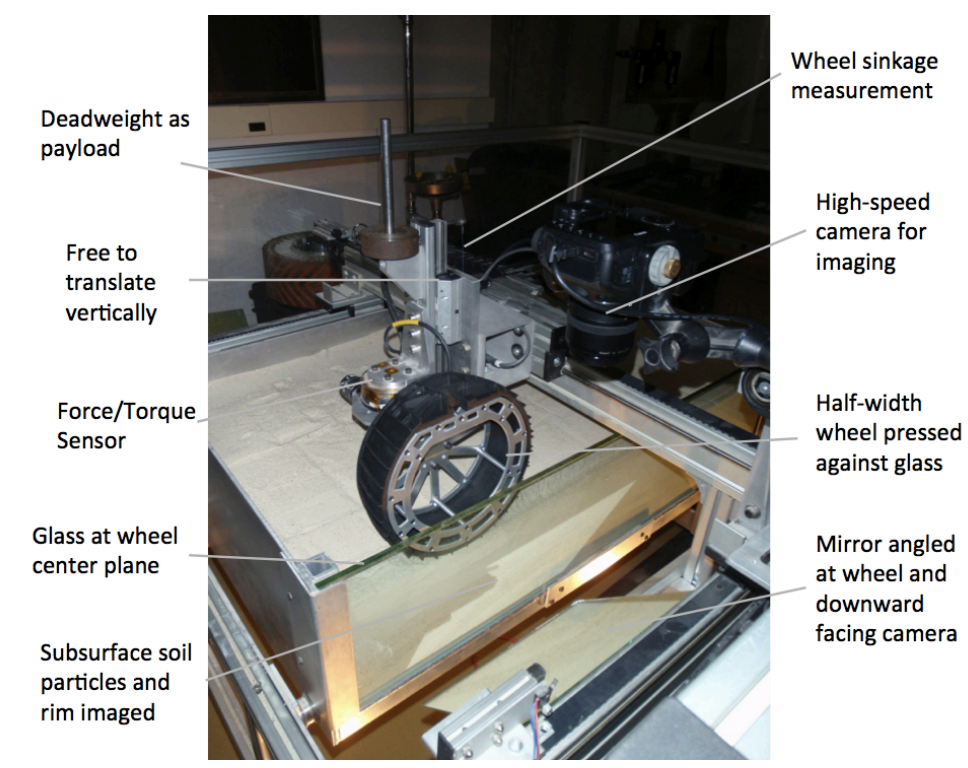

Fig 2: Wheel module, carriage and glass-walled soil bin. A $31 \mathrm{~cm}$ by $22 \mathrm{~cm}$ cross-section of soil below the wheel is imaged with a high-speed camera. 
The shearing analysis requires the ability to track soil motion. A digital SLR camera with a $50 \mathrm{~mm}$ macro lens was used to image the wheel-soil interface, logging frames simultaneously with the rest of the telemetry. A frame rate of 8 frames-per-second was used and is sufficiently fast for the slow speeds of wheel travel applied $(2 \mathrm{~cm} / \mathrm{sec})$. The camera is mounted perpendicular to the soil bin glass wall and travels with the wheel in the horizontal direction as the carriage moves. For most wheel specimens $(23$ to $50 \mathrm{~cm}$ ) a $31 \mathrm{~cm}$ wide by $22 \mathrm{~cm}$ high (soil depth) patch of soil is framed and able to capture the complete shear interface produced by the wheel in the regolith simulants utilized. External halogen flood lights at a high angle normal to the glass illuminate the soil particles.

Image processing comprises of optical flow and clustering techniques. The optical flow algorithm [Black96] tracks displacement of soil regions relative to a prior frame and calculates a motion vector at each pixel. Initial clustering separates each image into "soil" and "not soil" regions. Additional processing is continued only for "soil" regions. The magnitude of flow at each pixel of the soil regions is calculated from the optical flow vector fields. Soil flow is clustered into "significant" and "insignificant" magnitudes of motion. No explicit threshold is used to demarcate these clusters, but rather automatically adaptive clustering is used. The shear interface is derived from the boundary between significant and insignificant motions (Fig. 3). Soil flow direction is calculated from the optical flow vector fields, for soil regions exhibiting significant soil flow. Soil flow in any direction (360 degrees) is visualized, and an additional boundary is identified at points where the soil transitions between forward and rear flow. Figure 3 demonstrates sample output of the process, showing soil flow magnitude, shear interface between significant and insignificant flow, soil flow direction and boundary between forward and rear flow.

\section{$2.2 \quad$ Processed Results}

The soil displacement field plots generated during a single wheel experiment are used to identify the effect wheel parameters and design features have on performance. Performance metrics such as net thrust produced (referred to as drawbar pull [Bekker56]) are critical for evaluation but there little information provided that aids in investigation of the underlying principles governing the measured performance. As an example, parametric studies of grousered wheels are repeatedly encountered [Ding 11] [Bauer05]. To date, these investigations into the function of grousers has taken the approach of relying solely on performance measurements. Net traction, sinkage, slippage, wheel torques, power and reaction forces are typically measured during single wheel testing over a wide range of parameter changes, such as grouser spacing or height. Trends in the data are used to determine optimal parameter combination and conclusions sometimes inferred from these results. This approach is suitable for determining the response and performance of specific designs but provides limited information on the actual mechanisms and processes occurring in the soil that govern traction. Therefore, little knowledge 
can be gained in understanding how grousers function in general and how they should be implemented in design and vehicle operation.

Observing the soil shearing planes allows for qualitative analysis of how soil structure develops and react thrust loads or produces resistance to travel. The shear interface is indicative of the soil failure process and type. Analysis of this and the flows present within the soil can aid design of traction devices and study of terramechanic fundamentals. Figure 3 shows the processed results of a single wheel shear interface imaging analysis experiment. The Flow Velocity Magnitude and Flow Direction plots are used to analyze the wheel. These plots show processes typically present within the soil of a wheel operating in loose, granular soil.

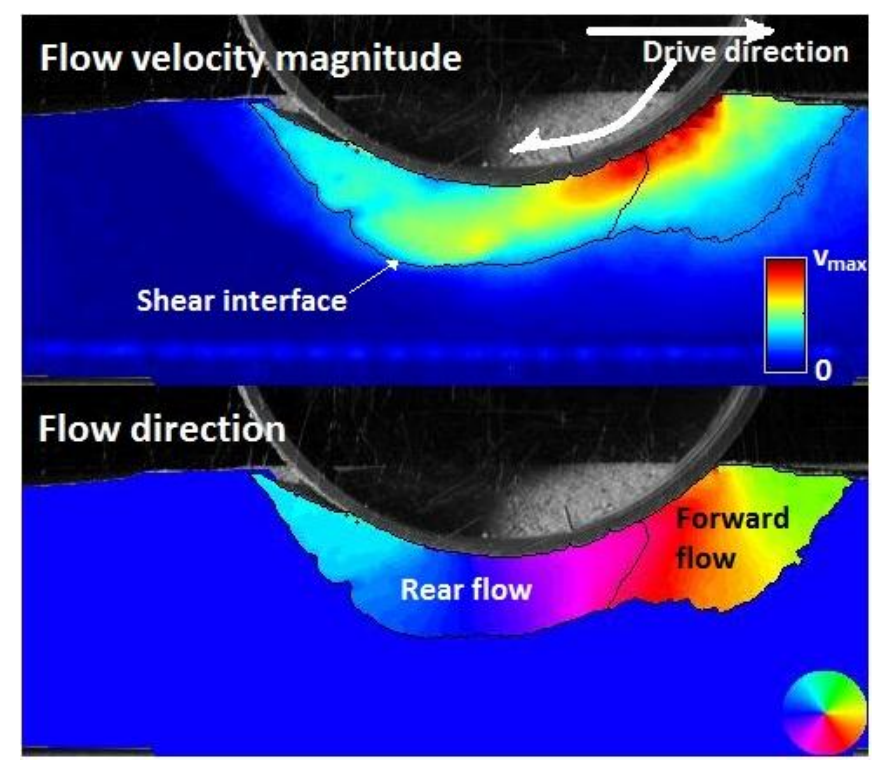

Fig 3: Shear Interface Imaging plots. Plots show soil flow magnitude (top) and direction (bottom). Magnitude is plotted from dark blue (stationary soil) to red (soil being displaced at highest speed). Direction (within the shear interface) is plotted as shown in the circular color legend. Note a convention of all plots are wheel travel left to right and at a $20 \%$ slip rate unless otherwise noted.

The flow velocity field plot uses the optical flow displacement field measured between image pairs and clustering methods for classification to display the soil flow speed. These plots (Fig. 3, top) scale from dark blue (stationary soil) to red (representing the soil flowing at maximum speed, $\mathrm{V}_{\max }$ ). This type of plot allows for the evaluation of the soil flow due to shearing. The shear interface is a key indicator of the means by which the wheel produces traction. This term, for purposes of this study, is defined as the region (line or band like in shape) where soil transitions from measured shear displacement (flowing) to near static (not flowing). 
The Flow Direction plot (Fig. 3 bottom) displays the direction of soil particle shearing as measured by the flow velocity field. The multi-colored wheel is the legend that maps color to direction with respect to the wheel coordinate frame. 'Dark blue' indicates soil particles moving completely horizontal in the left hand direction, opposite the direction of wheel travel. The direction of shearing aids in determining what type of soil failure process occurs, design features that may contribute to the failure and the identification of multiple flows, such as resistive types at the wheel front. The separation of two flows (Fig. 3, bottom), as detected by the developed analysis software, allows for the identification of forward flows and the measurement of the location of point of maximum shear stress along the rim. This occurs at the intersection of the wheel rim and flow separation point.

\subsection{General Experiment Considerations and Procedure}

As an example of this process, the experimentation of the wheel shown in Fig. 4 will be described. The single wheel imaging testbed is prepared with GRC-1 lunar soil simulant [Oravec10] before each test run. The soil is loosened to a state of lowest relative density and slightly compacted by use of a drop tamper method to produce repeatable soil properties. The wheel specimen shown in Fig. 4 is rigid, $23 \mathrm{~cm}$ diameter by $5.72 \mathrm{~cm}$ wide $(11.5 \mathrm{~cm}$ effective width), with the rim covered by course grain sandpaper. A 10kg payload is applied in the vertical direction. All experiments are analyzed at steady-state response of the soil and reaction loads. The test run begins at static sinkage and then travels under a controlled slip rate for approximately five wheel diameters in distance. All rigid wheels (rough rim or grousers) quickly entered steady-state sinkage, reaction loading and soil shearing behavior within the first wheel revolution. The testbed controls the wheel slip while maintaining a wheel tangential rim speed of $2 \mathrm{~cm} / \mathrm{sec}$ by varying horizontal travel (carriage) speed. Most experiments, such as shown in Fig. 4, are evaluated at $20 \%$ slip. Generally, wheel peak performance in loose, granular soil occurs between $10-30 \%$ slip and as such, $20 \%$ was chosen as a point of study for most evaluations. However, for each wheel configuration a full range of slip values (5-65\% slip, with 5\% slip intervals) are collected, but only key points such as $20 \%$ and $50 \%$ slip undergo soil shearing analysis. Additionally, for each experiment, at least three repeats were conducted.

The focus of this paper is to introduce the technique of improved shear interface imaging analysis (SIIA). An overview of the investigation of common wheel design features and resulting soil behavior through examples of experiments conducted will be utilized in the following section in effort to shed light on this technique. 


\section{Analysis Examples}

There is a set of important parameters commonly decided upon in the design of a wheel for planetary surface systems. Choices between a rigid or compliant rim, tread surface parameters such as rough or with grousers and wheel geometry such as diameter. These all have significant results on the soil behavior during operation of the vehicle and the resulting traction performance. The study of the effect of some of these parameters was conducted and preliminary results are shared.

To study the development of the net thrust produced by rigid wheels, soil shearing was investigated over a range of slip ratios, and over changes in wheel diameter, tread surface, and locomotion mode. The approach was to conduct a preliminary survey of design features that have effect on performance and attempt to associate soil shearing behavior with potential gains or losses.

\subsection{Rigid Wheel Soil Failure}

It is evident in Figure 11 that soil fails sharply at the shear interface and that there is significant forward flow. The shape of the shear interface, the point at which it originates at the rim (flow separation point) and magnitude of shearing indicate a forced type failure due to wheel rotation. This is further supported by the direction of the flow at the shear interface. For this wheel, the soil shearing direction is near vertical at the front of the wheel and returns to the surface behind the wheel at a near vertical direction. These types of behaviors are typical of small/medium diameter rigid wheels with mid-range performance where significant slip induced sinkage (slip-sinkage) is present. This leads to a high entrance angle. This type of observation of the soil behavior under a wheel rim is valuable in understanding terramechanic fundamentals and how a specific wheel design functions.

The degree of wheel slippage affects not only the net traction produced but also the state of soil behavior. Figure 12 shows the drawbar-slip curve for a $23 \mathrm{~cm}$ diameter $(5.72 \mathrm{~cm}$ wide) rigid wheel with a sand-paper like tread carrying a $10 \mathrm{~kg}$ payload. The shape of the curve is typical of most wheels in loose, granular soil relevant to planetary vehicles.

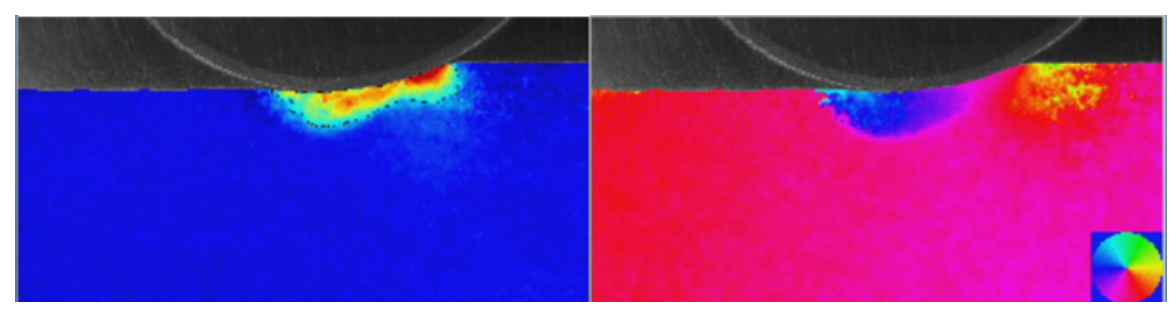

Fig 4: Plots shown are particle velocity magnitude (left) and direction (right). Unlike figure 3 , there is no threshold on the direction plot (right). The 'pink' soil in the bulk direction plot is stationary (except directly under the wheel). Compaction of soil in forward direction 
(yellow) is visible in front of leading edge (right) of wheel. This is evidence of forward motion resistance.

There are distinct changes of the soil behavior at key points of the drawbar-slip curve as seen in Figure 5. Three important observations can be made: (1) the shear interface size and shape does not change between 0.05 and 0.20 slip ratio although the drawbar pull produced quadrupled. (2) From 0.05 to 0.20 slip ratio, the soil shearing process within the shear interface transitions from a large gradient of shearing to near zero gradient within the shear interface (3) Lastly, above 0.20 slip ratio, the forward flow appears to diminish while the shearing zone (region within shear interface) begins to reduce in depth. There are a number of hypotheses that can be made from these observations. First, the shear interface of this wheel may be governed primarily by a mechanism other than applied thrust. This is evident since even at low slip and low load (0.05 slip and lower), the extent of the affected soil in shearing is similar to that at 0.20 slip ratio. It is possible that shearing induced by the rotation of the rim is excessive since large displacements must occur due to wheel sinkage (i.e. soil must be displaced downwards, rearwards, then back up). The second important hypothesis is that the knee in the drawbar-slip curve (about 0.20 slip in this example) may occur when the soil shearing is fully developed within the shear interface. Operating at a slip ratio above 0.20 appears to transition from a thrust generation type behavior of the wheel and soil, to an excavation behavior at increasing slip.

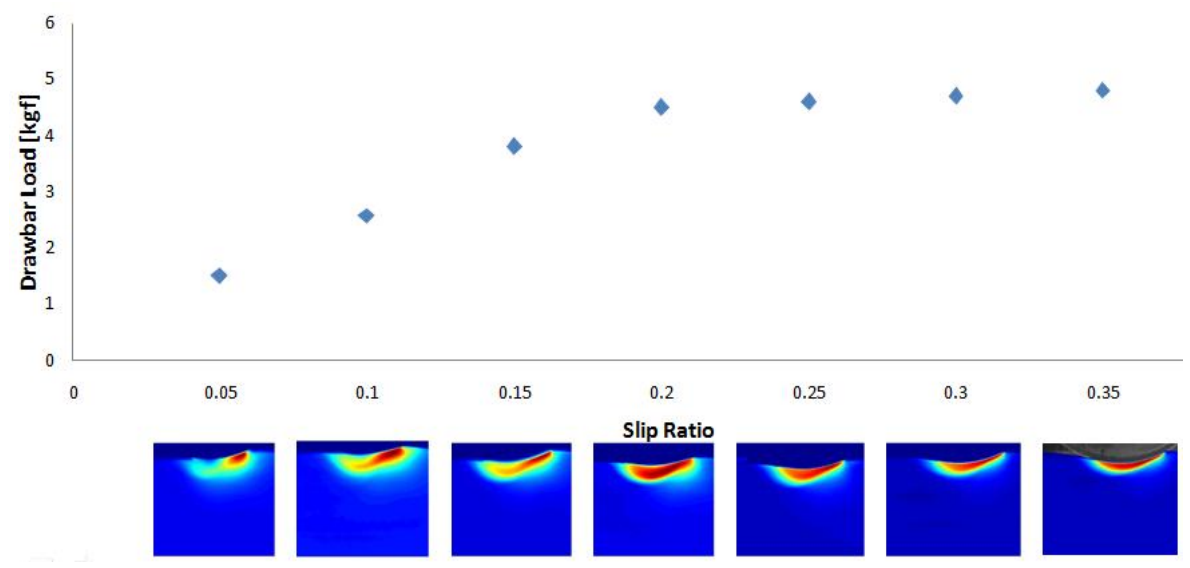

Fig 5: Drawbar-Slip curve with corresponding soil flow magnitude plots at 5\% slip intervals (see value above inset plots). Distinct changes in soil shearing behavior at key slip $(0.2)$ and load points are observed. 


\subsection{Walking}

To investigate the effect of the rotating rim on the generation of thrust, a study comparing walking locomotion to rolling locomotion was conducted. Push-roll like locomotion (use of walking and rolling wheels) has been demonstrated to produce high drawbar pull for increased locomotion capability [Moreland11b]. Utilizing shear interface imaging analysis, it was shown that the soil failure type of a walking wheel was different from a rigid rolling wheel (Fig. 6). This figure shows the "ground type failure" created by a walking wheel. This type of soil failure, due to the minute degree of shearing required, is able to produce multiple times the thrust of a rolling rigid wheel.

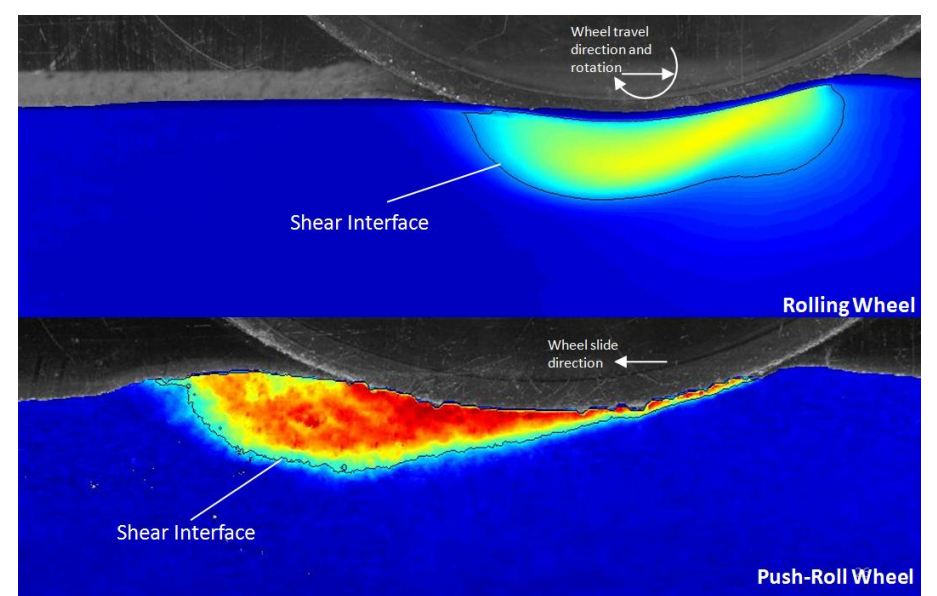

Fig 6: Shear interface analysis comparing rolling wheel to push-roll locomotion (walking wheel). "Ground type failure" of the soil is observed for the walking wheel, identifying a source of tractive gains.

\subsection{Wheel Diameter}

The diameter of a wheel has a profound effect on the behavior of the soil shearing and the resulting traction performance. An experiment comparing a $23 \mathrm{~cm}$ diameter rigid wheel to a $41 \mathrm{~cm}$ diameter rigid wheel of equal width, payload and slip is shown in Figure 7. Performance using the drawbar pull metric measured a $33 \%$ increase in traction for the larger diameter wheel. The behavior of the soil shearing is also observably different. The large diameter wheel lacks an observable forward flow and the soil shearing is in a near horizontal direction. Lowered resistive flows may not be the only contributor to the increase in measured drawbar pull. Soil shearing induced by the rim rotation is in the direction of travel (horizontal) and may account for the increased drawbar pull. The soil particles need not shear as much when only acted on horizontally. This creates a larger portion of the shear interface that can operate at the peak stress of the stress-strain curve of 
the soil (granular, cohesionless soil shear strength peaks at low shear strain). A lower shearing magnitude is also evident for the large diameter wheel.

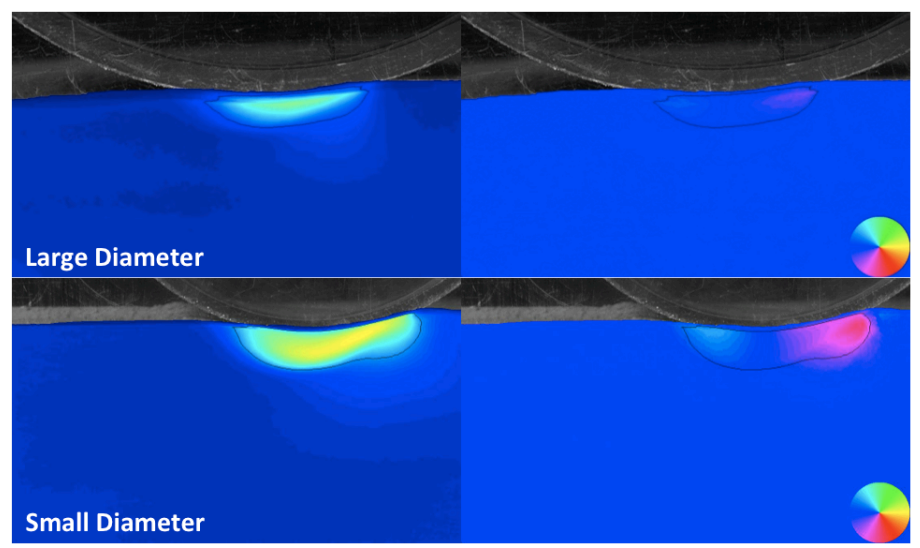

Fig 7: Variation of shear interface with change in wheel diameter. The large wheel shows nearly horizontal flow compared to large changes in flow direction (down then up) under the small wheel.

\subsection{Grousers}

Grousers are often employed in wheel designs for planetary rovers. The affect of soil shearing can be studied when analyzing these features. Figure 8 shows the periodic nature of soil shearing due to individual grouser effects. It appears that the grouser at the front of the wheel entering the soil have the greatest effect. Experiments with very close spaced grousers were also conducted and showed similar results but with periodicity proportional to spacing. As the optical flow algorithm utilizes overlapping image pairs, high fidelity movies of the grouser shearing effects can be utilized to observe individual grousers interacting with soil as the rim rotates.

The traction performance of the wheel without grousers (sand paper rim) and the same wheel with 48 grousers at $13 \mathrm{~mm}$ height act as extremes of the configurations and drawbar pull performance amongst those tested. Studying these two cases leads to an important realization. The soil displacement behavior of the grouserless wheel (Figure 4) and the 48 grouser wheel (Figure 9) are very different at the leading edge of the wheel. The Figure 4 direction plot, no grousers, shows a yellow patch of soil in front of the wheel that moves in a horizontal direction forward (low magnitude and within compaction regime). This is evidence of a significant motion resistance that would be reacted against the rim as soil is pushed forward and compacted downwards. This observed motion resistance would reduce the drawbar pull of the wheel. A wheel with high drawbar pull utilizing high performance grouser parameters (48 grousers at $13 \mathrm{~mm}$ height) however does not show evidence of a motion resistance. Additionally, a low resistance large diameter 
wheel did not have observable forward flow. It is reasonable to conclude from this observation that grousers can be implemented to significantly reduce compaction resistance and lead to higher drawbar pull. An increase in drawbar could also arise from an increase in thrust. Observations of soil shearing may also suggest whether the grousers provide additional thrust.

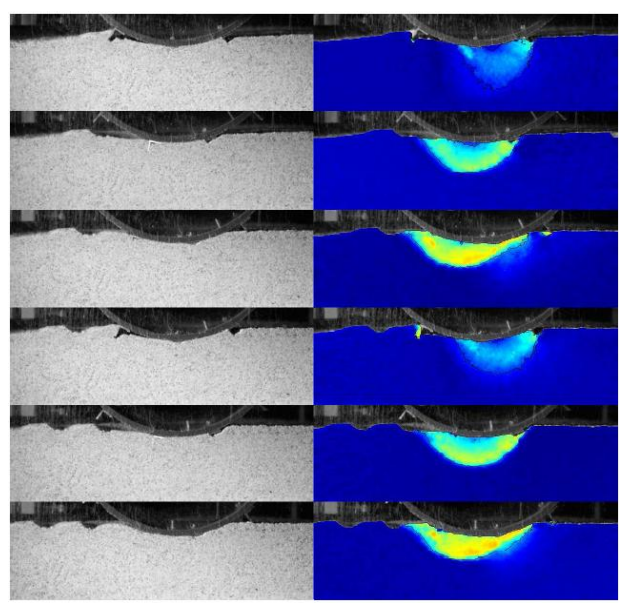

Fig 8: Time-lapse images (top to bottom) of soil shearing by a wheel with grousers over two cycles, two grouser plunges (flow magnitude plot shown). Distinct, periodic soil motion present as each new lug rotates into the soil. This occurs for even small grouser spacing's however the magnitude of soil shearing is lowered. Wheel travel is to the right.
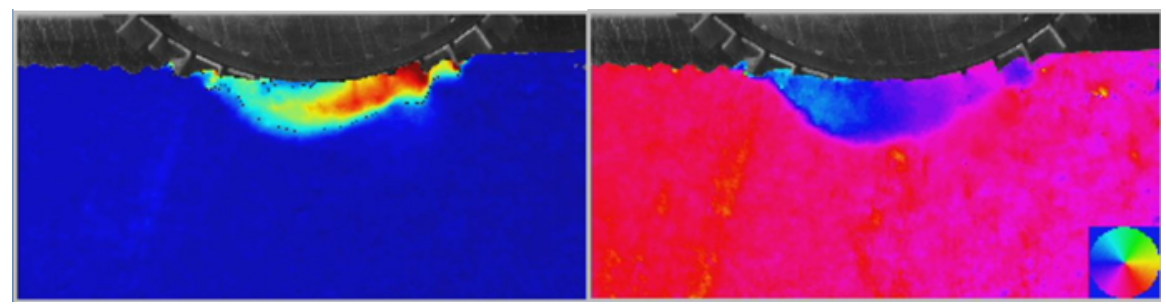

Fig 9: Rigid, grousered wheel SIIA. For a high drawbar grousered wheel (48lugs at $13 \mathrm{~mm}$ height), no observable evidence of forward compaction exists at the leading edge of the wheel. Therefore, little motion resistance should be present. Wheel motion to right.

The rear shear interface of grousered and non-grousered (wheels are quite similar (Figure 4 and 9 respectively). If it is assumed that in the rear flow region, the grousers are full of compacted soil, it will act like a wheel of larger diameter (only for the rear flow region, not front entrance area). With this assumption, the length, 
depth and shape of the shear interfaces of the two wheels are remarkably similar. If the magnitude of shearing and the soil density are similar, than the strength along the shear interface should be similar. The absolute shearing magnitude of the two wheels was measured to be similar using absolute particle velocity plots. Additionally, a reasonable assumption can be made that the soil density (as GRC1 is not highly compactable) in the rear flow region behind the wheel center would have undergone similar compaction do to wheel payload. For these reasons, the soil strength can be assumed to be similar. As the shear interface shape/size and soil strength are similar, it is reasonable to conclude that the thrust component of the drawbar pull are of similar value for both grousered and non-grousered wheels. Differences in thrust due to drastically different shear interfaces can cause high changes in thrust, however the failure modes are of the same type for both shear interface observed. As such, it is theorized that the gain in drawbar pull due to implementation of grousers arises solely from the reduction of compaction resistance

Single wheels test load cell measurements shows that wheels with grousers can be configured to increase drawbar pull. Compaction in the forward flow region in front of the contact area was not observed for wheels with grouser that generate high drawbar. Additionally, the shear interface of the region associated with generating thrust is similar for wheels with and without grousers. It is reasonable to conclude that increases in drawbar pull of wheels with grousers arises primarily from the decrease in compaction resistance. The increase in drawbar pull most likely is due to a decrease in motion resistance, not from an increase in thrust.

\subsection{Compliant Wheels}

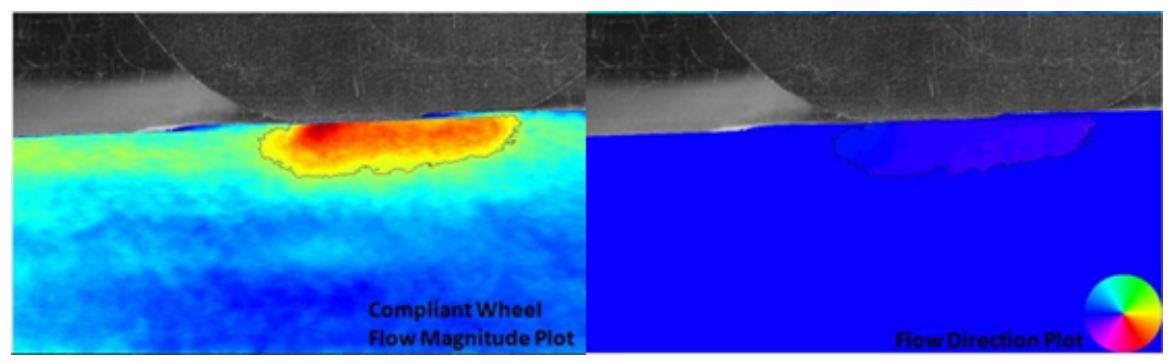

Fig 10: Compliant wheel shearing analysis showing low sinkage, no forward flow, low shearing magnitude and completely horizontal motion of soil displacement (may all be within compaction process).

Compliant wheels may provide large gains in performance for future surface exploration missions. Drawbar pull testing of individual wheels and full vehicles with compliant wheels has shown high tractive and energy performance. The study of the affect on soil behavior in the generation of thrust is essential. Experiments were conducted using a $23 \mathrm{~cm}$ diameter by $10 \mathrm{~cm}$ wide compliant wheel with 
sandpaper-like tread. The construction of the wheel did not produce uniform contact pressure as a pneumatic tire would, but a flat contact area was achieved. SIIA testing shows significant differences in soil behavior between the rigid wheel (Figure 4) and compliant type wheel (Figure 10). The direction of the soil displacement is completely horizontal. This may occur due to the extraordinary low sinkage and the flat shape of the contact along the length of the deformed rim.

It should be noted that the soil flow magnitude shown in Figure 10 is actually an order of magnitude lower than with the rigid wheel of Figure 4. As such, the soil shearing was so low; it appeared to be within the compaction regime of the soil simulant (as initial state was of low relative density). This limited study illustrates the importance of study the specific shear interface and soil behavior of a compliant wheel design when designing for high performance wheels. An understanding of how the observed behavior of the wheel-soil system affects performance will aid in developing more capable traction systems.

\section{Conclusion}

A technique for studying wheel-soil behavior and analyzing shear interfaces was developed and demonstrated. The technique can aid in design of high performance systems and increase the knowledge of terramechanics for wheels in a planetary environment. Examples investigating common wheel design parameters show a wide variation of shearing behavior that is intimately linked to traction performance.

The technique has been used for detailed quantitative observation of:

- Distinct changes in soil shearing behavior at key slip and load points

- Distinct failure modes beneath rolling and pushed wheels

- Variations in soil flow magnitude and direction between wheels of varying diameter and compliance

- Discrete periodic soil motions induced by grousers

- Compaction in the forward flow region in front of the contact area was not observed for wheels with grousers that generate high drawbar.

- Reduction of forward soil motion for grousers wheels correlated with grouser spacing/height that excavated at the leading edge enough to drastically lower the contact angle

The state of maturity of the measurement tool (software and hardware) is high enough that it can now used by designers of mobility platforms for loose, granular soil. Additionally, the technique provides the benefit of a different perspective on terramechanics fundamentals. 


\section{References}

[Bauer05] Bauer, R., W. Leung, and T. Barfoot. "Experimental and Simulation Results of Wheel-Soil Interaction for Planetary Rovers." IROS. 2005.

[Bekker48] Bekker, M. G. "Photographic method of determining the soil action beneath footings." Proc. 2nd Int. Conf. on Soil Mechanics and Foundation Engineering, Rotterdam, 193-194. 1948

[Bekker56] Bekker, M., "Theory of Land Locomotion,” The University of Michigan Press, Ann Arbor 1956

[Bekker60] Bekker, M. G. "Off-the-road locomotion”. University of Michigan Press, Ann Arbor. 1960

[Black96] Black, M. J. \& Anandan, P. "The robust estimation of multiple motions: Parametric and piecewise-smooth flow fields," CVIU, 63(1), pp. 75-104. 1996

[Ding11] Ding, L., H. Gao, Z. Deng, K. Nagatani, and K. Yoshida. "Experimental study and analysis on driving wheels' performance for planetary exploration rovers moving in deformable soil." Journal of Terramechanics, 2011: 27-45.

[Gachet03] Gachet, P. Klubertanz, G., et al,. "Interfacial Behavior of Unsaturated Soil with Small-scale Models and Use of Image Processing Techniques," GEOTECHNICAL TESTING JOURNAL,, 26, 12-21. 2003

[Harrison73] Harrison, W.L. "Soil failure under inclined loads-II." Journal of Terramechanics. 10(1), pp.11-50. 1973

[Khot07] Khot, L. R.; Salokhe, V. M.; Jayasuriya, H. \& Nakashima, H., "Experimental validation of distinct element simulation for dynamic wheel-soil interaction." Journal of Terramechanics, 44, 429 - 437, 2007

[Moreland11a] Moreland, S., Skonieczny, K., Wettergreen, D., Creager, C., and Asnani, V. "Soil Motion Analysis System for Examining Wheel-soil Shearing." Proc. 17 ${ }^{\text {th }}$ Int. Conf. of the International Society for Terrain-Vehicle Systems, Blacksburg September 2011

[Moreland11b] Moreland, S., Skonieczny, K., Wettergreen, D., Asnani, V., Creager, C., Oravec, H. "Inching Locomotion for Planetary Rover Mobility," IEEE Aerospace Conference, Big Sky, MT March 2011.

[Moreland12] Moreland, S., Skonieczny, K., Inotsume, H., and Wettergreen, D., "Soil Behavior of Wheels with Grousers for Planetary Rovers." IEEE Aerospace. Big Sky, MT: IEEE, 2012. 
[Oravec10] Oravec, H., V. Asnani, and X. Zeng. "Design and Characterization of GRC-1: A Soil for Lunar Terramechanics Testing in Earth-Ambient Conditions." Journal of Terramechanics, 2010: 361- 377 .

[Raschke96] Raschke, S,. Roman D., Hryciw \& Donohoe, G. W. "Microdeformations in Sands by Digital Image Processing and Analysis." Transportation Research Record, 1548, 31-37. 1996

[Wong66] Wong, J. Y., and Reece, A. R. "Soil failure beneath rigid wheels." Proc. $2^{\text {nd }}$ Int. Conf. of the International Society for Terrain-Vehicle Systems, Quebec City, 425-445. 1966

[Wong67] Wong, J. "Behavior of Soil Beneath Rigid Wheels.” Journal of Agricultural Engineering Research , vol 12(4) pp. 257-269. 1967 